\title{
ACCURATE AND EFFICIENT \\ RECONSTRUCTION OF DISCONTINUOUS FUNCTIONS FROM TRUNCATED SERIES EXPANSIONS
}

\author{
KNUT S. ECKHOFF
}

\begin{abstract}
Knowledge of a truncated Fourier series expansion for a discontinuous $2 \pi$-periodic function, or a truncated Chebyshev series expansion for a discontinuous nonperiodic function defined on the interval $[-1,1]$, is used in this paper to accurately and efficiently reconstruct the corresponding discontinuous function. First an algebraic equation of degree $M$ for the $M$ locations of discontinuities in each period for a periodic function, or in the interval $(-1,1)$ for a nonperiodic function, is constructed. The $M$ coefficients in that algebraic equation of degree $M$ are obtained by solving a linear algebraic system of equations determined by the coefficients in the known truncated expansion. By solving an additional linear algebraic system for the $M$ jumps of the function at the calculated discontinuity locations, we are able to reconstruct the discontinuous function as a linear combination of step functions and a continuous function.
\end{abstract}

\section{INTRODUCTION}

Spectral methods are in many cases known to work extremely well for problems involving smooth functions [4, 9]. For problems involving functions of finite regularity, however, and in particular for problems involving discontinuous functions, there are still a number of issues to be settled. In particular, it is well known that application of traditional spectral methods to nonlinear hyperbolic partial differential equations will often lead to numerical instabilities unless special measures are taken. The main reason for this is that the solutions of such equations may develop discontinuities (shocks) even for cases with smooth initial data.

The basic idea in spectral methods is to approximate all functions involved by truncated series expansions with respect to an a priori chosen sequence of functions. For problems involving periodic boundary conditions, truncated Fourier

Received by the editor October 3, 1991 and, in revised form, August 17, 1992.

1991 Mathematics Subject Classification. Primary 65M70, 65N35.

This paper is based on work done while the author was engaged at the SINTEF Multiphase Flow Laboratory, Trondheim, Norway. The research was supported by NTNF Contract No. OT45.23626 under the Program for Multiphase Flow Technology (PROFF) 1989-90. The support from The Royal Norwegian Council for Scientific and Industrial Research (NTNF), Amoco, Elf, Esso, Norsk Hydro, Saga, Shell, Statoil, Total, Aker Engineering, Framo Engineering, Norwegian Applied Technology, Kværner Engineering, and SINTEF is thankfully acknowledged. 
series expansions are widely used here, while truncated Chebyshev series expansions, as well as expansions in terms of various other types of Jacobi polynomials, are used for problems involving nonperiodic boundary conditions. The approximations obtained by such truncated series expansions for discontinuous functions are highly oscillatory, and the oscillations are particularly bad close to the discontinuities. This oscillatory behavior of the truncated series expansions near a discontinuity is known as the Gibbs phenomenon (at least for Fourier series) and could be regarded as the major cause of the problems connected with application of spectral methods to problems involving discontinuous functions.

Different ways of curing this deficiency of spectral methods have been suggested in the literature. Most of the suggested recipes are intended to eliminate, or at least considerably reduce, the oscillatory behavior of the truncated expansions by various ways of filtering. The idea is that more accurate approximations for the proper nonoscillatory discontinuous functions can be constructed that way. Traditionally, the problem has therefore been looked upon as the problem of designing a suitable filter for this purpose.

Many different ways of filtering have been suggested and studied in considerable detail in the literature cited in the reference list. Even though they often produce acceptable results, most of the suggested filtering techniques do not seem to take advantage of the full power of the spectral methods. At the same cost, they presently seem only, at best, to be able to produce results of accuracy comparable to those obtained by traditional finite difference methods for problems involving discontinuous functions.

The exceptions to this rule are the promising results obtained by utilizing step functions in the reconstruction of discontinuous functions, an approach which was iniiiated by Gottlieb et al. [8], and which has been further developed in $[1,2,3,7]$. That approach is in accordance with the idea introduced by Lax [12], namely that the oscillatory behavior obtained by a high-order method like a spectral method should contain enough information for us to be able to reconstruct the proper nonoscillatory discontinuous function by a postprocessing filter.

The idea of introducing step functions was originally only utilized as a cosmetic postprocessing operation $[1,2,7,8]$, intended to display the relevant physical results obtained by the calculations at a few preselected values of time. Throughout the integration of the governing partial differential equations in time, more traditional filtering was applied. Those filters, in that context, were only applied in order to stabilize the calculations, however, and not as in various other approaches to eliminate the Gibbs oscillations.

In the recent work [3] an approach is attempted where the integration process and the postprocessing are considered as a unity, with the intention to improve upon the results obtained by application of the underlying ideas in $[1,2,7,8]$. The results obtained in [3] are quite promising, but are in contrast to earlier works based on a reconstruction of the discontinuous functions at every timestep, utilizing step functions. In [3] only cases with at most one discontinuity present in each period are considered. For such cases the reconstruction is a relatively straightforward matter.

When there is more than one discontinuity present in each period, however, effective reconstruction of discontinuous functions utilizing step functions is no 
longer trivial, and may therefore easily turn out to be prohibitively expensive if one intends it to be applied often. In order to extensively apply such reconstructions, an efficient and accurate algorithm is therefore clearly needed. We have not seen published details of any such algorithm in the literature before. In this paper we shall therefore establish algorithms which are both accurate and efficient, and the reconstruction will be described both for truncated Fourier series expansions and for truncated Chebyshev series expansions. The algorithms will be based on the so-called Prony method [11, 16], which recently also has been utilized in various other application areas for Fourier analysis (see [14] and the references quoted there).

The algorithms for the reconstruction of discontinuous functions which we are going to describe in this paper are based on knowledge of accurate approximations for the exact Fourier or Chebyshev coefficients. If sufficient care is taken, however, the algorithms are still applicable in connection with the spectral collocation method, which therefore normally will be utilized for their practical implementation. The presentation will be given with such implementations in mind, and will therefore [4] involve mathematically unconventional, odd-looking, summation limits for the occurring truncated Fourier series.

\section{Fundamentals}

To a Riemann integrable complex-valued $2 \pi$-periodic function $u(x)$ we may associate a Fourier series

$$
u(x) \sim \sum_{k=-\infty}^{+\infty} \hat{u}_{k} e^{i k x}
$$

where

$$
\hat{u}_{k}=\frac{1}{2 \pi} \int_{-\pi}^{\pi} u(x) e^{-i k x} d x, \quad k=0, \pm 1, \pm 2, \ldots .
$$

For any given even integer $N>0$, we shall define the $N$ th-order truncated Fourier series associated with the $2 \pi$-periodic function $u(x)$ by

$$
P_{N} u(x)=\sum_{k=-N / 2+1}^{N / 2-1} \hat{u}_{k} e^{i k x} .
$$

As is well known $[9,18]$, the error involved when we approximate $u(x)$ by the truncated Fourier series expansion (3) depends strongly on the smoothness of the function $u(x)$. We shall in this paper limit our discussion to functions $u(x)$ which are piecewise smooth on $[-\pi, \pi]$. If we assume in addition that $u(x)$ is everywhere continuous and has continuous derivatives of order $p=$ $1,2, \ldots, m-1$, it can be shown $[9,18]$ that

$$
\hat{u}_{k}=O\left(|k|^{-(m+1)}\right) \text { as } k \rightarrow \pm \infty,
$$

and the best available global estimate is

$$
\max _{-\pi \leq x \leq \pi}\left|u(x)-P_{N} u(x)\right|=O\left(N^{-m}\right) \quad \text { as } N \rightarrow \infty .
$$


At a fixed point $x$ where $u^{(m)}(x)$ is continuous, however, it can be shown that

$$
u(x)-P_{N} u(x)=O\left(N^{-(m+1)}\right) \quad \text { as } N \rightarrow \infty .
$$

The above estimates give an important part of the explanation for why spectral methods work so well when all functions involved are smooth. When $u(x)$ is discontinuous, however, the estimates (4), (5), and (6) hold with $m=0$. As a global approximation for the function $u(x)$, (3) cannot therefore be expected to be particularly accurate. In fact, the Gibbs phenomenon involving large oscillations must be present, and it is known $[4,9]$ that the overshoot for the truncated Fourier series (3) is asymptotically equal to 0.08949 times the magnitude of the jump of the function at the discontinuity when $N \rightarrow \infty$.

One way of introducing the Chebyshev polynomials $T_{k}(x), k=0,1, \ldots$, is to define them on the interval $-1 \leq x \leq 1$ by the relations

$$
T_{k}(x)=\cos (k \theta), \quad \theta=\arccos x .
$$

Consider now an arbitrarily given complex-valued function $u(x)$ which is piecewise smooth on the interval $-1 \leq x \leq 1$. If we define $U(\theta)=u(\cos \theta)$, it then clearly follows that $U(\theta)$ is an even $2 \pi$-periodic function which is piecewise smooth on $-\pi \leq \theta \leq \pi$. Hence from (2) we get that $\widehat{U}_{-k}=\widehat{U}_{k}$, $k=1,2, \ldots$, in the Fourier series (1) associated with $U(\theta)$, which therefore can be written as a pointwise convergent Fourier cosine series

$$
\frac{1}{2}[U(\theta+)+U(\theta-)]=\sum_{k=0}^{\infty} \alpha_{k} \cos (k \theta),
$$

where the coefficients are given by $\alpha_{0}=\widehat{U}_{0}, \alpha_{k}=2 \widehat{U}_{k}, k=1,2, \ldots$.

From (7) it now follows by substitution of $\theta=\arccos x$ into (8) that for $-1<x<1$ we have

$$
\frac{1}{2}[u(x+)+u(x-)]=\sum_{k=0}^{\infty} \alpha_{k} T_{k}(x) .
$$

At the endpoints of the interval, $x=-1$ and $x=1$, it is not difficult to see that the sum of the Chebyshev series on the right-hand side in (9) must equal $u\left(-1^{+}\right)$and $u\left(1^{-}\right)$, respectively.

For any given integer $N>0$, we define the $N$ th-order truncated Chebyshev series associated with the function $u(x)$ by

$$
P_{N} u(x)=\sum_{k=0}^{N} \alpha_{k} T_{k}(x) .
$$

From the results we have referred to above for Fourier series, it is clear that the rate of convergence for Chebyshev series must depend strongly on the smoothness of the function $u(x)$. If in addition to the assumption of piecewise smoothness we also assume that $u(x)$ is continuous and has continuous derivatives of order $p=1,2, \ldots, m-1$ for $-1<x<1$, it can be readily seen that $U(\theta)=u(\cos \theta)$ is everywhere continuous and has continuous derivatives of order $p=1,2, \ldots, m-1$ with respect to the variable $\theta$. Hence, it follows 
from (4) that in this case

$$
\alpha_{k}=O\left(k^{-(m+1)}\right) \quad \text { as } k \rightarrow \infty .
$$

Similarly, it follows from the above assumptions and (5) that

$$
\max _{-1 \leq x \leq 1}\left|u(x)-P_{N} u(x)\right|=O\left(N^{-m}\right) \quad \text { as } N \rightarrow \infty .
$$

Finally, it follows from the above assumptions and (6) that if we let $x$ be a fixed point in $[-1,1]$ where $u^{(m)}(x)$ is continuous, then we have that

$$
u(x)-P_{N} u(x)=O\left(N^{-(m+1)}\right) \quad \text { as } N \rightarrow \infty .
$$

\section{RECONSTRUCTION FROM TRUNCATED FOURIER SERIES}

Now let $u(x)$ be a $2 \pi$-periodic function which is piecewise smooth on $[-\pi, \pi]$. Suppose that $u(x)$ is known to be discontinuous, but that we do not know the actual form of the function $u(x)$. Suppose, however, that we do know the $N$ th-order truncated Fourier series (3) associated with the function $u(x)$ for some $N$; i.e., assume that the numbers $\hat{u}_{k}$ are known for $k=0, \pm 1, \pm 2, \ldots, \pm(N / 2-1)$.

The latter assumption may at first glance seem to be of limited value since we know from the results referred to in the previous section that the truncated Fourier series (3) associated with a discontinuous function $u(x)$ must be highly oscillatory and therefore does not readily provide any accurate approximation for $u(x)$. The problem we would like to address then, is the problem of determining a good approximation for the function $u(x)$ solely from knowledge of the Fourier coefficients $\hat{u}_{k}$ for $k=0, \pm 1, \pm 2, \ldots, \pm(N / 2-1)$.

A prototype $2 \pi$-periodic function which is piecewise smooth on $[-\pi, \pi]$, and which has one discontinuity in each period, is given by the $2 \pi$-periodic extension of the function

$$
V(x ; \beta)= \begin{cases}\frac{1}{2 \pi}(\beta-\pi-x) & \text { if }-\pi<x<\beta, \\ \frac{1}{2 \pi}(\beta+\pi-x) & \text { if } \beta<x \leq \pi,\end{cases}
$$

where $\beta$ is a parameter at our disposal such that $-\pi \leq \beta<\pi$. The step function (or sawtooth function) (14) has a jump discontinuity of magnitude +1 at $x=\beta .^{1}$ The Fourier coefficients (2) associated with (14) are given by

$$
\widehat{V}_{0}(\beta)=0, \quad \widehat{V}_{k}(\beta)=\frac{1}{2 \pi i k} e^{-i k \beta}, \quad k= \pm 1, \pm 2, \ldots
$$

The assumption that the function $u(x)$ is piecewise smooth on $[-\pi, \pi] \mathrm{im}$ plies that $u(x)$ may have a finite number, $M$ say, of jump discontinuities on $[-\pi, \pi]$, but that $u(x)$ is otherwise smooth. Thus the assumption that the discontinuous $2 \pi$-periodic function $u(x)$ is piecewise smooth on $[-\pi, \pi]$ is clearly equivalent to the assumption that $u(x)$ can be written in the following

\footnotetext{
${ }^{1}$ In this paper we introduce the convention that the function values are disregarded at the points where a piecewise smooth function is discontinuous.
} 
way for some integer $M \geq 1$ :

$$
u(x)=v(x)+\sum_{j=1}^{M} A_{j} V\left(x ; \gamma_{j}\right),
$$

where $v(x)$ is some continuous $2 \pi$-periodic function which is piecewise smooth on $[-\pi, \pi], V\left(x ; \gamma_{j}\right)$ is given by (14) with $\beta=\gamma_{j}$, and $A_{j}, \gamma_{j}$ are some constants for $j=1, \ldots, M$.

Clearly, the points $x=\gamma_{j}, j=1, \ldots, M$, are the locations of the discontinuities for the function $u(x)$ given by $(16)$ in the period $[-\pi, \pi)$, and $A_{j}$ are the associated jumps. The problem we are now going to study is the problem of determining good approximations for the function $v(x)$ and the constants $A_{j}, \gamma_{j}$ in (16) solely from knowledge of the numbers $\hat{u}_{k}$ for $k=0, \pm 1, \pm 2, \ldots, \pm(N / 2-1)$. In the following we shall assume that the integer $M \geq 1$ is known.

From (15) it follows that the Fourier coefficients (2) associated with (16) are given by $\hat{u}_{0}=\hat{v}_{0}$ and

$$
\hat{u}_{k}=\hat{v}_{k}+\frac{1}{2 \pi i k} \sum_{j=1}^{M} A_{j} e^{-i k \gamma_{j}}, \quad k= \pm 1, \pm 2, \ldots
$$

If we introduce the notation

$$
C_{k}=2 \pi i k\left(\hat{u}_{k}-\hat{v}_{k}\right), \quad k= \pm 1, \pm 2, \ldots,
$$

then (17) can be written in the following way:

$$
\sum_{j=1}^{M} A_{j} e^{-i k \gamma_{j}}=C_{k}, \quad k= \pm 1, \pm 2, \ldots
$$

If we now seek an algebraic equation of degree $M$,

$$
z^{M}+X_{1} z^{M-1}+X_{2} z^{M-2}+\cdots+X_{M-1} z+X_{M}=0,
$$

with roots $z_{j}=e^{-i \gamma_{j}}, j=1, \ldots, M$, we find as a consequence of (19) and Prony's method [16] (as presented in [11] or [14]) that the coefficients $X_{j}, j=$ $1, \ldots, M$, in (20) must satisfy the following inhomogeneous linear algebraic equation:

$$
C_{k}+C_{k-1} X_{1}+C_{k-2} X_{2}+\cdots+C_{k-M} X_{M}=0
$$

for every integer $k \neq 0,1,2, \ldots, M$.

Unfortunately, the coefficients $C_{k}, \ldots, C_{k-M}$ in (21) are not a priori known, since by (18) they depend on the unknown function $v(x)$. By assumption, however, the function $v(x)$ in $(16)$ is continuous and piecewise smooth on $[-\pi, \pi]$, hence by (4) we have

$$
\hat{v}_{k}=O\left(|k|^{-2}\right) \text { as } k \rightarrow \pm \infty .
$$

On the other hand, with $M \geq 1$, the function $u(x)$ is discontinuous, and consequently by (4) we have

$$
\hat{u}_{k}=O\left(|k|^{-1}\right) \text { as } k \rightarrow \pm \infty .
$$


It is also well known [18] that the decay of the Fourier coefficients $\hat{u}_{k}$ as $k \rightarrow \pm \infty$ is not faster than that shown in (23) for discontinuous $2 \pi$-periodic functions $u(x)$ which are piecewise smooth on $[-\pi, \pi]$.

It is the difference in the asymptotic behavior of the Fourier coefficients associated with a discontinuous function (23), compared to the behavior of the coefficients for a continuous function (22), which now forms the basis for our reconstruction of discontinuous functions of the form (16) from the known truncated Fourier series. In fact, from the above estimates we get as $k \rightarrow \pm \infty$

$$
\widetilde{C}_{k} \stackrel{\text { def }}{=} 2 \pi i k \hat{u}_{k}=O(1), \quad 2 \pi i k \hat{v}_{k}=O\left(|k|^{-1}\right) .
$$

Consequently, from (18) it follows that

$$
C_{k}=\widetilde{C}_{k}+O\left(|k|^{-1}\right) \quad \text { as } k \rightarrow \pm \infty,
$$

and for $|k|$ sufficiently large we can therefore in equation (21) approximate $C_{k}$ by $\widetilde{C}_{k}$. We note that the quantities $\widetilde{C}_{k}$ are directly determined by the known Fourier coefficients $\hat{u}_{k}$ by (24). Furthermore, we note that the error involved by that approximation depends on the actual smoothness of the function $v(x)$, and may therefore in practice be much more favorable than the estimate given in (25).

Usually any given set of $M$ equations (21), obtained for $M$ different values of $k$, will be linearly independent if $M$ is the correct number of discontinuities in each period for the function $u(x)$. In special cases, however, it can be seen that there is a slight possibility that the obtained system may be linearly dependent. It is for example a straightforward calculation to show from (19) for the case $M=2$ that the determinant associated with the pair of equations (21) obtained for the two different values $k=p$ and $k=q$ is given by

$$
A_{1} A_{2}\left(e^{i \gamma_{1}}-e^{i \gamma_{2}}\right)\left[e^{-i\left(p \gamma_{1}+q \gamma_{2}\right)}-e^{-i\left(q \gamma_{1}+p \gamma_{2}\right)}\right] \text {. }
$$

Thus, as expected, the pair of equations (21) obtained for $k=p$ and $k=q$ will usually be linearly independent if $A_{1} \neq 0, A_{2} \neq 0$, and $e^{i \gamma_{1}} \neq e^{i \gamma_{2}}$, i.e., if we really have $M=2$. In fact, the only possibility that the expression (26) can vanish when $M=2$ is that we have

$$
(p-q)\left(\gamma_{1}-\gamma_{2}\right)=2 n \pi
$$

for some $n=0, \pm 1, \pm 2, \pm 3, \ldots$ Unless $p=q \pm 1$, (27) can clearly be satisfied for special values of $\gamma_{1} \neq \gamma_{2}$. For the general case where the function $u(x)$ has exactly $M$ discontinuities in each period, it is not difficult to show from (19) that we will always obtain a linearly independent system if we choose a system (21) corresponding to $M$ consecutive values of $k$.

Summing up the above discussion, we may conclude that an approximate equation (20) for the $M$ discontinuity locations will be obtained if the coefficients $X_{j}, j=1, \ldots, M$, are calculated by the linear system of algebraic equations

$$
\widetilde{C}_{k}+\widetilde{C}_{k-1} X_{1}+\widetilde{C}_{k-2} X_{2}+\cdots+\widetilde{C}_{k-M} X_{M}=0,
$$

for $k=N / 2-M, N / 2-M+1, \ldots, N / 2-1$, when $N>4 M$. With the solution $X_{j}, j=1, \ldots, M$, of (28) substituted, we then solve (20). From the 
$M$ roots $z_{j}, j=1, \ldots, M$, thus found, we calculate the uniquely determined approximations for the $M$ discontinuity locations $\gamma_{j}, j=1, \ldots, M$, on the interval $[-\pi, \pi)$ by the equations

$$
e^{-i \gamma_{j}}=\frac{z_{j}}{\left|z_{j}\right|}, \quad j=1, \ldots, M .
$$

Next, approximations for the $M$ discontinuity jumps $A_{j}, j=1, \ldots, M$, are calculated by the following linear system of $M$ algebraic equations obtained from (19) and (25):

$$
\sum_{j=1}^{M} A_{j} e^{-i k \gamma_{j}}=\tilde{C}_{k}, \quad k=N / 2-M, N / 2-M+1, \ldots, N / 2-1 .
$$

Finally, an approximate function $v(x)$ in (16) is determined by an $N$ thorder truncated Fourier series (3) with Fourier coefficients $\hat{v}_{k}$ given by

$$
\hat{v}_{k}=\hat{u}_{k}-\frac{1}{2 \pi i k} \sum_{j=1}^{M} A_{j} e^{-i k \gamma_{j}}
$$

for $k= \pm 1, \pm 2, \ldots, \pm(N / 2-1)$, while $\hat{v}_{0}=\hat{u}_{0}$.

This completes the reconstruction of the discontinuous $2 \pi$-periodic function $u(x)$ of the form (16). We would like to add, however, that it is possible to improve upon the above reconstruction (i.e., make it more robust and more accurate) if we also take into consideration the alternative equations obtained for $k$ negative ( $|k|$ should still be chosen as large as possible). The resulting overdetermined systems of properly weighted linear algebraic equations can for instance be handled by the least squares method [6]. We shall not consider such modifications further here, but only mention that the most obvious improvements will be seen in the determination of the $M$ discontinuity jumps $A_{j}$, $j=1, \ldots, M$.

\section{TRuncated Chebyshev Series}

Now let $u(x)$ be an arbitrarily given piecewise smooth function on the interval $[-1,1]$. Suppose furthermore that $u(x)$ is known to be discontinuous, but that we do not know the actual form of the function $u(x)$. Suppose, however, that we do know the $N$ th-order truncated Chebyshev series (10) associated with it for some $N$; i.e., the numbers $\alpha_{k}$ associated with the function $u(x)$ are assumed to be known for $k=0,1,2, \ldots, N$. The problem we would like to address in this section is the problem of determining a good approximation for the function $u(x)$ solely from knowledge of those Chebyshev coefficients $\alpha_{k}$ in an analogous way as we did for Fourier series in the preceding section.

From the discussion in $\S 2$ and the above assumptions it clearly follows that for each discontinuity of the function $u(x)$ in the interval $(-1,1)$, the associated $2 \pi$-periodic even function $U(\theta)=u(\cos \theta)$ is discontinuous at two different points in the interval $(-\pi, \pi)$ lying symmetric with respect to $\theta=0$ and with jumps of opposite signs, but with the same magnitude, at those two points. From the discussion in $\S 2$ we furthermore know that the function $U(\theta)$ must be piecewise smooth on $-\pi \leq \theta \leq \pi$, and that the Fourier series (1) associated 
with $U(\theta)$ can be written as the pointwise convergent Fourier cosine series (8) which is directly obtained from the Chebyshev series for $u(x)$.

From the given $N$ th-order truncated Chebyshev series expansion (10) associated with the function $u(x)$, the $(2 N+2)$ th-order truncated Fourier series expansion (3) for the associated even function $U(\theta)$ can therefore readily be set up. If $M$ is the number of discontinuities for the function $u(x)$ on the interval $(-1,1)$, we may consequently apply the method described in the preceding section in order to reconstruct the function $U(\theta)$ with $2 M$ discontinuities on the interval $(-\pi, \pi)$ in the form

$$
U(\theta)=\Phi(\theta)+\sum_{j=1}^{M} A_{j}\left[V\left(\theta ;-a_{j}\right)-V\left(\theta ; a_{j}\right)\right],
$$

where $\Phi(\theta)$ is some continuous $2 \pi$-periodic even function which is piecewise smooth on $[-\pi, \pi], V\left(\theta ; \pm a_{j}\right)$ is given by (14), and $A_{j}, a_{j}$ are some constants for $j=1, \ldots, M$. By introducing the substitution $\theta=\arccos x$ into (32), we then obtain a reconstruction for the function $u(x)$ on the interval $[-1,1]$.

The above procedure for reconstruction of discontinuous functions from truncated Chebyshev series expansions is both accurate and quite efficient in practical applications. We do note, however, that in the procedure we actually calculate each discontinuity location twice. In fact, $a_{j}$ and $-a_{j}$ are calculated as two different discontinuity locations although they both correspond to the same discontinuity location $x=\cos a_{j}$ for the function $u(x)$. It may therefore be worthwhile to look for a more direct approach to the reconstruction of the function $u(x)$ in this case.

From (32) we see that a natural prototype $2 \pi$-periodic even function which is piecewise smooth on $[-\pi, \pi]$ and which has two discontinuities in each period, is given by the function

$$
W(\theta ; a)=V(\theta ;-a)-V(\theta ; a),
$$

where $V(\theta ; \pm a)$ is given by (14), and where $a$ is a parameter at our disposal such that $0<a<\pi$. From (14) and (33) it readily follows that

$$
W(\theta ; a)= \begin{cases}-\frac{1}{\pi} a & \text { if }-\pi<\theta<-a, \\ 1-\frac{1}{\pi} a & \text { if }-a<\theta<a, \\ -\frac{1}{\pi} a & \text { if } a<\theta \leq \pi,\end{cases}
$$

and from (15) it is not difficult to show that the Fourier series for the function $W(\theta ; a)$ is given by

$$
\frac{1}{2}[W(\theta+; a)+W(\theta-; a)]=\frac{2}{\pi} \sum_{k=1}^{\infty} \frac{\sin (k a)}{k} \cos (k \theta) .
$$

Let us now, for the time being, suppose that the given piecewise smooth function $u(x)$ is known to be discontinuous with only one discontinuity in 
the interval $(-1,1)$. The associated even function $U(\theta)$ is then discontinuous with two discontinuities in each perioci. This is clearly equivalent to the assumption that $U(\theta)$ can be written as

$$
U(\theta)=\Phi(\theta)+A W(\theta ; a),
$$

where $\Phi(\theta)$ is some continuous $2 \pi$-periodic even function which is piecewise smooth on $[-\pi, \pi], W(\theta ; a)$ is given by (34), and $A, a$ are some constants. From (34), (36) it is clear that $\theta=-a$ and $\theta=a$ are the locations for the two discontinuities of $U(\theta)$ on the interval $(-\pi, \pi)$, and $A$ is the magnitude of the jumps of $U(\theta)$ at those two points.

If we introduce the substitution $\theta=\arccos x$ into (36), we obtain for $-1 \leq$ $x \leq 1$

$$
u(x)=\phi(x)+A w(x ; a),
$$

where $u(x)$ is the given discontinuous function, $\phi(x)$ is the continuous function which is uniquely defined on the interval $[-1,1]$ by the requirement that $\phi(\cos \theta)=\Phi(\theta)$, and $w(x ; a)$ is the discontinuous function which is uniquely defined on the interval $[-1,1]$ by the requirement that $w(\cos \theta ; a)=$ $W(\theta ; a)$. It is readily seen from (34) that

$$
w(x ; a)= \begin{cases}-\frac{1}{\pi} a & \text { if }-1 \leq x<\cos a, \\ 1-\frac{1}{\pi} a & \text { if } \cos a<x \leq 1 .\end{cases}
$$

From the discussion in $\S 2$ and (24) it follows that

$$
\widetilde{C}_{k}=-\widetilde{C}_{-k}=2 \pi i k \widehat{U}_{k}=\pi i k \alpha_{k}, \quad k=1,2, \ldots .
$$

As a consequence, it is easily seen that the system of equations (28) obtained for $M=2$ with $k=N$ and $k=-N+2$ always has the solution $X_{2}=1$, which actually is the exact solution since $X_{2}=e^{-i \gamma_{1}} e^{-i \gamma_{2}}$ by (20) and $\gamma_{1}=-a, \gamma_{2}=a$ for the case considered here. Assuming that $C_{N-1} \neq 0$, we furthermore obtain from (28), (39) that

$$
\cos a=-\frac{X_{1}}{2} \approx \frac{\widetilde{C}_{N}+\widetilde{C}_{N-2}}{2 \widetilde{C}_{N-1}}=\frac{N \alpha_{N}+(N-2) \alpha_{N-2}}{2(N-1) \alpha_{N-1}},
$$

which is easily found to be in accordance with the two roots of the equation (20) for $M=2$. Thus, from (37), (38) it is therefore clear that (40) gives an approximate location for the discontinuity of the function $u(x)$ on the interval $[-1,1]$ when $u(x)$ has only one discontinuity.

As we shall show in the following, it is possible in a similar way to arrive at simplified approximate equations for the locations of the discontinuities for an arbitrarily given piecewise smooth function $u(x)$ defined on $[-1,1]$ by a suitable modification of the approach described in the preceding section. Let us therefore now assume that we know the Chebyshev coefficients $\alpha_{k}$ for $k=$ $0,1,2, \ldots, N$ for some sufficiently large $N$ for the discontinuous function $u(x)$, but that we do not know the actual form of $u(x)$.

The assumption that the piecewise smooth function $u(x)$ is discontinuous on $(-1,1)$ is clearly equivalent to the assumption that $u(x)$ can be written in 
the following way for some integer $M \geq 1$ :

$$
u(x)=\phi(x)+\sum_{j=1}^{M} A_{j} w\left(x ; a_{j}\right),
$$

where $\phi(x)$ is some piecewise smooth function which is continuous on $[-1,1]$, $w\left(x ; a_{j}\right)$ is given by $(38)$, and $A_{j}, a_{j}$ are some constants for $j=1, \ldots, M$. If we introduce the substitution $x=\cos \theta$ into (41) and apply the notation introduced earlier in this section, we obtain

$$
U(\theta)=\Phi(\theta)+\sum_{j=1}^{M} A_{j} W\left(\theta ; a_{j}\right) .
$$

The Fourier coefficients (2) associated with (42) are in view of (35) given by

$$
\widehat{U}_{k}=\widehat{U}_{-k}=\frac{\alpha_{k}}{2}=\widehat{\Phi}_{k}+\frac{1}{\pi k} \sum_{j=1}^{M} A_{j} \sin \left(k a_{j}\right), \quad k=1,2, \ldots,
$$

and $\widehat{U}_{0}=\widehat{\Phi}_{0}=\alpha_{0}$. If we introduce the notation

$$
E_{k}=\pi k\left(\widehat{U}_{k}-\widehat{\Phi}_{k}\right), \quad k=1,2, \ldots,
$$

then (43) can be written in the following way:

$$
\sum_{j=1}^{M} A_{j} \sin \left(k a_{j}\right)=E_{k}, \quad k=1,2, \ldots
$$

The function $\phi(x)$ in (41) is assumed to be continuous and piecewise smooth on $[-1,1]$, hence by $(11)$ we have

$$
\widehat{\Phi}_{k}=O\left(|k|^{-2}\right) \text { as } k \rightarrow \infty .
$$

With $M \geq 1$, the function $u(x)$ is discontinuous, hence by (11),

$$
\widehat{U}_{k}=O\left(|k|^{-1}\right) \text { as } k \rightarrow \infty .
$$

As in the preceding section, it is this difference in behavior (47) of the Fourier coefficients associated with a discontinuous function, compared to the behavior (46) of the coefficients for a continuous function, which forms the basis for the reconstruction of discontinuous functions of the form (41) from the known truncated Chebyshev series. In fact, for $k$ sufficiently large, we can in view of the above estimates expect that

$$
E_{k} \approx \pi k \widehat{U}_{k}=\frac{\pi k \alpha_{k}}{2} \stackrel{\text { def }}{=} \widetilde{E}_{k}=O(1) \quad \text { as } k \rightarrow \infty .
$$

This together with (45) gives us approximate equations for the quantities $A_{j}$ and $a_{j}$. If we consider (45), (48) for a sufficient number of sufficiently large values of $k$, we may therefore be able to determine approximate values for $A_{j}$ and $a_{j}$ for $j=1, \ldots, M$. We shall now describe how this can be most economically done in practice. 
For $M=1$ a direct calculation for (45) easily shows that the following identity holds for every $k=2,3, \ldots$ :

$$
2 E_{k} \cos a=E_{k+1}+E_{k-1},
$$

which clearly gives the approximate equation (40) for the location of the discontinuity when the approximation (48) is introduced. In order to avoid problems connected with the possible exceptional case where $E_{N-1}=0$, and thus get a robust algorithm, we may here calculate the location of the discontinuity $x=\cos a$ as the least squares solution [6] of the following two linear algebraic equations:

$$
2 \widetilde{E}_{N-1} \cos a=\widetilde{E}_{N}+\widetilde{E}_{N-2}, \quad 2 \widetilde{E}_{N-2} \cos a=\widetilde{E}_{N-1}+\widetilde{E}_{N-3} .
$$

For $M=2$ direct calculations for (45) similarly show that the following identities hold:

$$
\begin{aligned}
& E_{k}=A_{1} \sin \left(k a_{1}\right)+A_{2} \sin \left(k a_{2}\right), \quad k=1,2,3, \ldots, \\
& Q_{k} \stackrel{\text { def }}{=} \frac{1}{2}\left(E_{k+1}+E_{k-1}\right) \\
& =A_{1} \sin \left(k a_{1}\right) \cos a_{1}+A_{2} \sin \left(k a_{2}\right) \cos a_{2}, \quad k=2,3,4, \ldots, \\
& \text { (53) } R_{k} \stackrel{\text { def }}{=} \frac{1}{2}\left(Q_{k+1}+Q_{k-1}\right) \\
& =A_{1} \sin \left(k a_{1}\right) \cos ^{2} a_{1}+A_{2} \sin \left(k a_{2}\right) \cos ^{2} a_{2}, \quad k=3,4,5, \ldots .
\end{aligned}
$$

From (51), (52), and (53) it now easily follows that for $k=3,4,5, \ldots$

$$
E_{k} \cos a_{1} \cos a_{2}-Q_{k}\left[\cos a_{1}+\cos a_{2}\right]+R_{k}=0 .
$$

This equation (54) plays essentially the same role here as the equation (21) played in the preceding section for the case $M=2$. In fact, if we seek an algebraic equation of degree 2 ,

$$
z^{2}+X z+Y=0,
$$

with roots $z_{1}=\cos a_{1}$ and $z_{2}=\cos a_{2}$, we see from (54) that the coefficients $X, Y$ in (55) must satisfy the inhomogeneous linear algebraic equation

$$
E_{k} Y+Q_{k} X+R_{k}=0
$$

for every $k=3,4,5, \ldots$.

Usually, but unfortunately not always, a given pair of equations (56) for $X, Y$, obtained for two consecutive values of $k=3,4,5, \ldots$, will be linearly independent if we really have $M=2$. In order to avoid the possible linear dependence for the exceptional cases and thus get a robust algorithm, we have found it necessary to consider the enlarged, usually overdetermined system of four equations (56), obtained for four consecutive values of $k=3,4,5, \ldots$. In view of (48), we therefore see that an approximate equation for the two discontinuity locations (55) will be obtained if the coefficients $X, Y$ are determined as the least squares solution [6] of the following linear system of algebraic 
equations:

$$
\widetilde{E}_{k} Y+\widetilde{Q}_{k} X+\widetilde{R}_{k}=0, \quad k=N-2, N-3, N-4, N-5,
$$

where in accordance with (48), (52), and (53) we have introduced the notation

$$
\widetilde{Q}_{k} \stackrel{\text { def }}{=} \frac{1}{2}\left(\widetilde{E}_{k+1}+\widetilde{E}_{k-1}\right), \quad \widetilde{R}_{k} \stackrel{\text { def }}{=} \frac{1}{2}\left(\widetilde{Q}_{k+1}+\widetilde{Q}_{k-1}\right) .
$$

With the solution $X, Y$ of (57) substituted, we then solve (55). The two roots $z_{1}, z_{2}$ thus found, are then the uniquely determined approximations for the two discontinuity locations $x_{1}=\cos a_{1}, x_{2}=\cos a_{2}$ in the interval $(-1,1)$ for the function $u(x)$. From (48), (58) we see that the eight largest availalle Chebyshev coefficients $\alpha_{N-7}, \ldots, \alpha_{N}$ are utilized in order to set up the equations (55), (57) for the determination of those two discontinuity locations.

In a similar way we expect that by utilizing the $4 M$ largest available Chebyshev coefficients, it is possible to arrive at an algebraic equation of degree $M$ for the $M$ discontinuity locations for the arbitrarily given piecewise smooth function $u(x)$ on $[-1,1]$. Since the procedure for this construction should be clear from the above discussion, we restrict ourselves in the following to describing it for the case where $M=3$, and introduce in addition to (48), (58) the notation

$$
\widetilde{S}_{k} \stackrel{\text { def }}{=} \frac{1}{2}\left(\widetilde{R}_{k+1}+\widetilde{R}_{k-1}\right) \text {. }
$$

The quantities $X, Y, Z$ are then determined as the least squares solution [6] of the following linear system of algebraic equations:

$$
\widetilde{E}_{k} Z+\widetilde{Q}_{k} Y+\widetilde{R}_{k} X+\widetilde{S}_{k}=0
$$

for $k=N-3, N-4, \ldots, N-8$, and this solution is substituted into the equation

$$
z^{3}+X z^{2}+Y z+Z=0
$$

The three roots $z_{1}, z_{2}, z_{3}$ of equation (61) are then the uniquely determined approximations for the three discontinuity locations $x_{1}=\cos a_{1}, x_{2}=\cos a_{2}$, $x_{3}=\cos a_{3}$ for the function $u(x)$ on the interval $(-1,1)$.

When the $M$ approximate discontinuity locations for the arbitrarily given piecewise smooth function $u(x)$ have been determined on $(-1,1)$ by the procedure described above, the associated values of the quantities $a_{1}, \ldots, a_{M}$ are uniquely determined on the interval $(0, \pi)$. Hence $(45),(48)$ give the following approximate, overdetermined, linear system of algebraic equations for the jumps $A_{1}, \ldots, A_{M}$ :

$$
\sum_{j=1}^{M} A_{j} \sin \left(k a_{j}\right)=\widetilde{E}_{k}, \quad k=N-2 M+1, \ldots, N,
$$

for which we again determine the least squares solution [6]. Finally, an approximate function $\phi(x)$ in (41) is given by an $N$ th-order truncated Chebyshev 
series (10) with Chebyshev coefficients $\zeta_{k}$ given by

$$
\zeta_{k}=2 \widehat{\Phi}_{k}=\alpha_{k}-\frac{2}{\pi k} \sum_{j=1}^{M} A_{j} \sin \left(k a_{j}\right), \quad k=1,2, \ldots,
$$

and $\zeta_{0}=\widehat{\Phi}_{0}=\alpha_{0}$. This completes the reconstruction of the given discontinuous function $u(x)$ of the form (41).

\section{NUMERICAL RESULTS}

The implementation of the algorithms described in the preceding sections has not involved special difficulties. We have utilized LINPACK subroutines [5] in order to solve the occurring linear systems of algebraic equations, including the least squares problems. For the determination of the discontinuity locations, the roots of the governing polynomials $(20),(55),(61)$, etc. have been calculated by the Laguerre method, where we have been able to use the subroutines provided in [15]. We have found the routines applied both accurate and efficient. The only modification we found profitable in addition to converting the Laguerre routines from [15] to double precision and changing the tolerances accordingly, was to change the default starting guess for the roots when solving (20). The reason for this is simply that the roots of $(20)$ which we are concerned with, are lying on, or close to, the unit circle in the complex plane, hence the default starting guess 0 does not give priority to any of the actual roots of (20), and is therefore not suitable in our case.

When the considered function is a linear combination of a set of step functions (14) obtained for different values of the parameter $\beta$, the reconstruction of the function from its Fourier coefficients by the method described in $\S 3$ should according to the given theory be exact. This is confirmed by our numerical experiments where such functions have been reconstructed with essentially machine accuracy. In particular, for piecewise constant functions, we have found in our double-precision PC-calculations ( 53 bits) that in absolute value, the error in the calculated discontinuity locations usually is less than $10^{-14}$ and that the error in the calculated jumps usually is less than $10^{-13}$.

Often the accuracy is better than the figures stated above, and in a few cases which we are going to look at in more detail below, the accuracy is less favorable. The change in accuracy of the calculated discontinuity locations which we have observed when we increase the number of discontinuities in each period, $M$, has been very modest (we have considered up to 30 discontinuities in each period). A slight decrease in accuracy of the reconstructed function can be observed, however, when the jumps of the function at the various discontinuity locations are several orders of magnitude different in size. We have also observed a slight decrease in accuracy of the calculated jumps when we increase the number of terms in the truncated Fourier series, $N$, while the accuracy of the calculated discontinuity locations in that case seems less affected.

The only situation where we have found significant changes in the accuracy for the reconstruction of piecewise constant functions, is when we have clustering of the discontinuity locations. In fact, when two or more discontinuities are located too close to each other, the errors introduced by the reconstruction can be much larger than the figures mentioned above. If, for example, for the 
case $N=128$ and $M$ up to 30 , we consider functions where the distance between two of the discontinuities is only 0.0001 , while the other discontinuity locations are more evenly distributed, we have found that in absolute value the error in the calculated clustered discontinuity locations typically can be $10^{-7}$, while the accuracy of the other discontinuities is not seriously affected. In some cases where the other discontinuity locations are far from evenly distributed, we have found that the calculated discontinuity locations may become even less accurate. If, for example, we let three of the discontinuities be clustered with distance 0.001 , the clustered discontinuities are often not accurately recovered at all in our calculations. Clearly, it is the precision employed in the calculations which is the important limiting factor here. It is interesting to note, however, that even in the latter cases we have not observed any drastic change in accuracy for the other, unclustered discontinuities, neither for the calculated locations, nor for the calculated jumps.

If we now turn to periodic functions which can be expressed as the sum of a smooth function and a piecewise constant function, our expectations of spectral accuracy for the reconstruction described in $\S 3$ is confirmed by our numerical calculations; i.e., the error decays faster than any power of $N^{-1}$ as $N$ increases. As an illustration, we shall consider the function

$$
f(x)=p(x)+\frac{3}{5-4 \cos x},
$$

where $p(x)$ is a piecewise constant function. We shall consider two different examples; in case (I) we let $p(x)$ be the $2 \pi$-periodic extension of the following function with three discontinuities in each period:

$$
p(x)= \begin{cases}0 & \text { if } \quad 0.0<x<1.0 \\ 2 & \text { if } 1.0<x<2.5 \\ -1 & \text { if } 2.5<x<3.0 \\ 0 & \text { if } 3.0<x \leq 2 \pi\end{cases}
$$

and in case (II) we let $p(x)$ be the $2 \pi$-periodic extension of the following function with seven discontinuities in each period:

$$
p(x)=\left\{\begin{array}{lll}
0 & \text { if } & 0.0<x<1.0 \\
2 & \text { if } & 1.0<x<2.5 \\
-1 & \text { if } & 2.5<x<3.0 \\
1 & \text { if } & 3.0<x<4.0 \\
-1 & \text { if } & 4.0<x<4.7 \\
-2 & \text { if } & 4.7<x<5.0 \\
-1 & \text { if } & 5.0<x<5.4 \\
0 & \text { if } & 5.4<x \leq 2 \pi
\end{array}\right.
$$


The absolute value of the largest error in the calculated discontinuity locations and the associated jumps for different values of $N$ were in our numerical calculations found to be as follows for these two cases:

\begin{tabular}{|c|c|c|c|c|}
\hline$N$ & 32 & 64 & 128 & 256 \\
\hline \hline Location-error (I) & 0.158 & $0.733 \cdot 10^{-5}$ & $0.266 \cdot 10^{-14}$ & $0.311 \cdot 10^{-14}$ \\
Jump-error (I) & 1.48 & $0.159 \cdot 10^{-4}$ & $0.124 \cdot 10^{-13}$ & $0.107 \cdot 10^{-13}$ \\
\hline Location-error (II) & 1.50 & 0.00479 & $0.316 \cdot 10^{-11}$ & $0.808 \cdot 10^{-13}$ \\
Jump-error (II) & 5.00 & 0.0131 & $0.613 \cdot 10^{-11}$ & $0.270 \cdot 10^{-12}$ \\
\hline
\end{tabular}

The spectral accuracy for the described method of reconstruction should be quite evident from the above table. Clearly, roundoff errors are becoming dominant for the largest values of $N$ shown for the considered functions (64).

Finally, let us consider the least favorable case where also the derivative of the function in question has jumps at the discontinuity locations. The accuracy we can expect for the reconstruction described in $\S 3$ for that case can be illustrated by considering the $2 \pi$-periodic extension of the following piecewise polynomial function with five discontinuities in each period:

$$
f(x)=\left\{\begin{array}{lll}
2+x-4 x^{2} & \text { if } & 1.0<x<1.5 \\
-5+x+x^{2} & \text { if } & 1.5<x<2.5 \\
2-3 x+2 x^{2} & \text { if } & 2.5<x<3.5 \\
1-2 x+x^{2} & \text { if } & 3.5<x<5.0 \\
40+x-x^{2} & \text { if } & 5.0<x<2 \pi+1.0
\end{array}\right.
$$

The absolute value of the largest error in the calculated discontinuity locations and the associated jumps for the function (67) for different values of $N$ were found to be as follows:

\begin{tabular}{|c|c|c|c|c|c|}
\hline$N$ & 32 & 64 & 128 & 256 & 512 \\
\hline \hline Location-error & 0.0764 & 0.00581 & 0.00123 & 0.000320 & 0.0000677 \\
Jump-error & 1.49 & 0.153 & 0.0315 & 0.00846 & 0.00168 \\
\hline \hline $2 \pi / N$ & 0.196 & 0.0982 & 0.0491 & 0.0245 & 0.0123 \\
\hline
\end{tabular}

The results given for the function (67) in the above table could be regarded as representative for the many functions we have looked at of this type. Often more accurate results are obtained for the lowest values of $N$, and in a few cases with large variations in jump-sizes and/or clustering of discontinuity locations, we have seen less favorable results. For the largest values of $N$ the above results are representative for all cases we have looked at except those with extreme clustering of discontinuity locations. For comparison we have in the table also given the corresponding spacing $2 \pi / N$ between neighboring collocation points. For all values of $N$ shown, we see that the locations of the discontinuities are determined with subgrid accuracy. Since this is an example of the least favorable case for the method described in $\S 3$, it should be fair to conclude 
that the method is quite accurate. Even without caring about optimization of the code, our calculations for the most time-consuming reconstructions we have looked at $(M=30, N=1024)$ are done in a matter of seconds on a PC; it should therefore also be fair to conclude that the method is efficient.

Although our numerical experiments for the Chebyshev case have been less extensive than for the Fourier case, the algorithms described in $\S 4$ for the reconstruction of discontinuous functions from their truncated Chebyshev expansions have been seen to give completely analogous numerical results in all cases we have looked at. We therefore strongly believe that the numerical results we have presented above for the Fourier case also are representative for the Chebyshev case. We shall therefore not consider further numerical results here.

\section{Discussion}

We have in this paper studied properties of truncated Fourier series expansions for discontinuous $2 \pi$-periodic functions, and truncated Chebyshev series expansions for discontinuous nonperiodic functions defined on the interval $[-1,1]$. In both cases we have found that it is possible to construct an algebraic equation of degree $M$ for the $M$ locations of the discontinuities in one period for a periodic function, and in the interval $(-1,1)$ for a nonperiodic function. The $M$ coefficients in that algebraic equation of degree $M$ are obtained by solving a linear algebraic system of equations determined by the coefficients in the known truncated expansion. By solving an additional linear algebraic system of equations for the $M$ jumps of the function at the calculated discontinuity locations, we are able to reconstruct the discontinuous function as a linear combination of step functions and a continuous function.

In the described reconstruction of discontinuous functions, the number of discontinuities, $M$, has been assumed to be known. For some applications, however, $M$ may not be known a priori, and therefore has to be determined as part of the reconstruction. For such cases the described algorithms give us several options for actually determining the correct number $M$. In fact, if for the Fourier case we pick a possible trial number, $\widetilde{M}$ say, of discontinuities in each period and then try by the described algorithm to look for the coefficients in the corresponding algebraic equation (20) of degree $\widetilde{M}$ for the locations of those discontinuities, the exact linear system of $\widetilde{M}$ equations (21) will be linearly dependent if $\widetilde{M}$ is larger than the actual number of discontinuities $M$. Thus the determinant of the matrix in the approximate system (28) will be close to zero for such cases. If the trial number $\widetilde{M}$ on the other hand is smaller than $M$, we may by the described algorithms be able to calculate the roots $z_{1}, \ldots, z_{\tilde{M}}$ of the corresponding algebraic equation (20) of degree $\widetilde{M}$ for the locations of those discontinuities. For such cases, however, the modulus of those roots $\left|z_{1}\right|, \ldots,\left|z_{\tilde{M}}\right|$ will most likely be far away from 1 (at least one of them will probably always be clearly different from 1). Analogous observations can be made for the Chebyshev case; it therefore seems relatively simple to design algorithms for the determination of the actual number of discontinuities $M$ from the known truncated series expansion in cases where it is not known a priori. The design of the most efficient algorithm for that purpose will clearly 
depend on what additional information can be inferred from the actual application considered. We would also like to remark that further details on possible algorithms for the determination of the correct number of discontinuities $M$ can be inferred from the discussion given in [14].

The accuracy and rate of convergence for the described reconstruction of the discontinuous function depends primarily on the smoothness of the continuous part of the reconstructed function. That continuous part is necessarily piecewise smooth by the introduced assumptions, but not necessarily more regular than that in general. If the continuous part is smooth, the reconstruction has been found to actually involve subgrid modelling of spectral accuracy as the number of terms $N \rightarrow \infty$. On the extreme other hand, if the derivative of the continuous part of the reconstructed discontinuous function is discontinuous, we cannot expect the method of reconstruction to be better than of the order $O\left(N^{-1}\right)$ as $N \rightarrow \infty$. In our numerical experiments for the latter case, however, we have found that the locations of the discontinuities typically are determined with an accuracy of the order $O\left(N^{-2}\right)$, which for $N$ sufficiently large is clearly better than just somewhere between two adjacent nodes.

As indicated above, the accuracy of the described method of reconstruction of discontinuous functions is least favorable when the continuous part of the reconstructed discontinuous function is not continuously differentiable. For such cases it is possible to improve upon the accuracy by utilizing not only step functions, but additional classes of special functions in the reconstruction, as briefly indicated in [3]. Although such modified reconstructions can be obtained by algorithms of a similar structure as those described in the present paper, the computations involved will be more expensive. For applications where the improvement in accuracy can account for the extra cost in the calculations, however, such modified, more accurate, reconstructions can be useful. We therefore intend to discuss such modified reconstructions in more detail in a follow-up paper in the near future.

\section{BIBLIOGRAPHY}

1. S. Abarbanel and D. Gottlieb, Information content in spectral calculations, Progress in Scientific Computing, Vol. 6 (Proc. U.S.-Israel Workshop, 1984) (E. M. Murman and S. S. Abarbanel, eds.), Birkhäuser, Boston, 1985, pp. 345-356.

2. S. Abarbanel, D. Gottlieb, and E. Tadmor, Spectral methods for discontinuous problems, NASA-CR-177974, ICASE Report no. 85-38, also in Numerical methods for fluid dynamics II, Proc. Conf. (Reading, 1985) (K. W. Morton and M. J. Baines, eds.), Clarendon Press, Oxford, 1986, pp. 129-153.

3. W. Cai, D. Gottlieb, and C.-W. Shu, Essentially nonoscillatory spectral Fourier methods for shock wave calculations, Math. Comp. 52 (1989), 389-410.

4. C. Canuto, M. Y. Hussaini, A. Quarteroni, and T. A. Zang, Spectral methods in fluid dynamics, Springer-Verlag, New York, 1988.

5. J. J. Dongarry, J. R. Bunch, C. B. Moler, and G. W. Stewart, LINPACK user's guide, SIAM, Philadelphia, PA, 1979.

6. G. H. Golub and C. F. Van Loan, Matrix computations, John Hopkins Univ. Press, Baltimore, MD, 1983.

7. D. Gottlieb, Spectral methods for compressible flow problems, Proc. 9th Internat. Conf. Numer. Methods Fluid Dynamics (Saclay, France, 1984) (Soubbaramayer and J. P. Boujot, eds.), Lecture Notes in Phys., vol. 218, Springer-Verlag, Berlin and New York, 1985, pp. 48-61. 
8. D. Gottlieb, L. Lustman, and S. A. Orszag, Spectral calculations of one-dimensional inviscid compressible flows, SIAM J. Sci. Statist. Comput. 2 (1981), pp. 296-310.

9. D. Gottlieb, and S. A. Orszag, Numerical analysis of spectral methods: Theory and applications, SIAM, Philadelphia, PA, 1977.

10. D. Gottlieb and E. Tadmor, Recovering pointwise values of discontinuous data within spectral accuracy, Progress in Scientific Computing, Vol. 6 (Proc. U.S.-Israel Workshop, 1984) (E. M. Murman and S. S. Abarbanel, eds.), Birkhäuser, Boston, 1985, pp. 357-375.

11. F. B. Hildebrand, Introduction to numerical analysis, 2nd ed., Tata McGraw-Hill, New Delhi, 1974.

12. P. D. Lax, Accuracy and resolution in the computation of solutions of linear and nonlinear equations, Recent Advances in Numerical Analysis, Proc. Symposium Univ. of WisconsinMadison (C. de Boor and G. H. Golub, eds.), Academic Press, New York, 1978, pp. 107-117.

13. A. Majda, J. McDonough, and S. Osher, The Fourier method for nonsmooth initial data, Math. Comp. 32 (1978), 1041-1081.

14. G. Majda, W. A. Strauss, and M. Wei, Computation of exponentials in transient data, IEEE Trans. Antennas and Propagation 37 (1989), 1284-1290.

15. W. H. Press, B. P. Flannery, S. A. Teukolsky, and W. T. Vetterling, NUMERICAL RECIPES, the art of scientific computing, Cambridge Univ. Press, Cambridge, 1989.

16. R. Prony, Essai expérimental et analytique ... , J. L'Ecole Polytech. (Paris) 1 (1795), cahier 2, 24-76.

17. E. Tadmor, Convergence of spectral methods for nonlinear conservation laws, SIAM J. Numer. Anal. 26 (1989), 30-44.

18. A. Zygmund, Trigonometric series, Vol I., Cambridge Univ. Press, Cambridge, 1968.

Department of Mathematics, University of Bergen, Allegt. 55, N-5007 Bergen, NORWAY

E-mail address: reske@mi.uib.no 